\title{
Why support a separate medical access framework for cannabis?
}

\author{
Elizabeth A. Cairns BSc, Melanie E.M. Kelly PhD
}

Cite as: CMAJ 2017 July 17;189:E927-8. doi: 10.1503/cmaj.170427

M

edical access to cannabis was first granted in Canada in 1999, with several amendments leading to the current Access to Cannabis for Medical Purposes Regulations. ${ }^{1}$ Patients have the right to access cannabis for medical purposes if they have the support of a health care practitioner. Three cannabinoid-based drugs are also approved for use in Canada: dronabinol and nabilone for chemotherapy-induced nausea and vomiting, and nabiximols for spasticity and neuropathic pain in multiple sclerosis, and cancer pain.

On Dec. 13, 2016, the Task Force on Cannabis Legalization and Regulation, appointed by the Government of Canada, released its recommendations on a framework for the legalization and regulation of cannabis in Canada, including the continued access to medical cannabis and cannabinoid-based drugs in a stream separate from recreational access. ${ }^{2}$ In response, the Canadian Medical Association (CMA) stated that it is not in support of the recommendation for two separate streams, citing concerns over lack of clinical evidence and guidance for medical use. $^{3}$ We recognize the substantial gaps in knowledge surrounding cannabinoid-based therapeutics; however, as experienced researchers in the field, we support the task force's recommendations and framework to maintain a separate medical stream, as set out within the current draft of the "Cannabis Act." Additional recognition by the CMA and other professional health care associations would validate the recommendations by the task force and would ensure continued support for this framework through development of this bill.

Recreational cannabis and cannabinoid-based drugs (including medical cannabis) are not equivalent. Without a program that supports medical use, patients may lose access to supports that the Federal Court of Canada has deemed appropriate, ${ }^{1}$ which fosters safe and appropriate use. ${ }^{2}$ Furthermore, having only one stream continues to fuel the stigma surrounding cannabinoid-based therapeutics and delegitimizes patients who state that they use these drugs out of necessity rather than choice. ${ }^{2}$

Historically, cannabis has been used for the treatment of various ailments. ${ }^{5}$ However, modern cannabinoid research is still a relatively young field; discovery of the human endocannabinoid system has occurred mainly within the last three decades. In large part, this is due to the complexity of the cannabis plant. Initial investigation was difficult, given the variability between chemical varieties and grow-

\section{KEY POINTS}

- Recreational and medical cannabis are not equivalent and, therefore, should have different frameworks for access.

- Although there are still substantial gaps in knowledge surrounding medical use, a separate medical framework provides an essential route to inform future policy decisions regarding the health of Canadians.

- This separate framework would support patients and health care providers by helping to reduce stigma, provide standardized systems for ongoing patient monitoring and facilitate continued education for health care providers.

- A separate framework would also provide incentives to strengthen research beyond harms reduction, including research into efficacy and safety of use, as well as to drive development of novel cannabinoid-based therapeutics that are eligible for drug identification numbers.

ing conditions, and methods of delivery. Of the over 400 chemical entities found in cannabis to date, scientists have characterized the pharmacology of only a few, and still have an incomplete understanding of how these cannabinoids function when combined. ${ }^{6}$ Use of newer synthetic cannabinoids has also provided challenges, often because of off-target effects at previously unknown or uncharacterized receptors. ${ }^{7,8}$ In addition, our understanding of the changes in the endocannabinoid system in pathologic states is still incomplete. ${ }^{9}$ To answer these complex questions, much more research must be done. However, aside from the regulatory hurdles required to acquire cannabinoids for study, negative perceptions associated with basic and clinical cannabinoid research continue to hamper research progress and have, in the past, restricted funding availability for cannabinoid research. ${ }^{10}$

In this context, hesitation from health care practitioners is understandable. Clearly, more research is needed to provide better guidelines about use. However, lack of evidence is not often a result of negative data, but it occurs because the research has yet to be carried out in large enough or properly controlled clinical studies. ${ }^{5}$ The type of research that is needed to address these unanswered questions will not occur or will be more difficult to justify if cannabinoid-based therapeutics continue to be dismissed without full consideration. ${ }^{10}$ 
There is substantial evidence that supports use of cannabinoid-based therapeutics for muscle spasticity, chronic pain, and lessening chemotherapy-induced nausea and vomiting, and moderate evidence supporting these therapeutics as sleep aids in several secondary sleep disturbances. ${ }^{5}$ However, there is a large body of low-quality and anecdotal evidence showing effective use of cannabinoids in the treatment of various other disorders, such as reducing symptoms of anxiety, posttraumatic stress disorder and Tourette syndrome, after other conventional treatments have failed. ${ }^{5,6}$ More research is required in some of these areas to strengthen efficacy evidence; however, existing data indicate important therapeutic possibilities.

Continuing with a separate supported medical stream after legalization would reduce risk to patients; this would enable informed decisions as to whether or not a cannabinoid-based treatment is the most appropriate option for their health needs. Maintaining a separate medical stream for cannabis would also provide additional incentives for continued studies that further knowledge about the endocannabinoid system and its potential as a therapeutic target, outside of harms reduction. As recommended by the task force, these projects could be funded through revenue from the taxed sale of cannabis, possibly through priority-status granting schemes by the Canadian Institutes of Health Research. Further research in this area can provide clearer answers on safety and efficacy, and lead to the development of appropriate cannabinoid-based pharmaceuticals with drug identification numbers.

A separate medical stream would also support infrastructure to provide increased and continued education for health care practitioners on the latest advances in cannabinoid research, including physicians, pharmacists and nurse practitioners, all of whom could work in tandem to ensure maximal monitoring and education of patients.

Maintaining a separate medical stream is not without costs and administrative hurdles; however, it is our opinion that these costs would be outweighed by the substantial benefits resulting from medical research and education that would help to shape future health policies. For example, patients would be able to have informed discussions with nurse practitioners or physicians about how a cannabinoid-based approach may or may not be appropriate for them given their medical history, and pharmacists could provide information about different forms of delivery or formulations. Physicians and nurse practitioners could provide ongoing monitoring that involves screening the patient for efficacy, with dose and formulation adjustment if necessary, and also risk factors of abuse.

Having a separate medical stream does not mean an endorsement of cannabinoid-based therapeutics as a cure-all. It is about providing the infrastructure to make informed choices for the patient and health care practitioner, and prioritizing a path to more concrete answers. The recommendations by the task force about maintaining a separate medical stream provide the best route to ensure greater patient safety and effective disease treatment.

\section{References}

1. Access to cannabis for medical purposes regulations, SOR/2016-230. Ottawa: Minister of Justice; 2017.

2. Task Force on Cannabis Legalization and Regulation. A framework for the legalization and regulation of cannabis in Canada. Ottawa: Health Canada; 2016.

3. CMA statement on report of Task Force on Cannabis Legalization and Regulation. Ottawa: Canadian Medical Association; 2016. Available: https://www.cma.ca/En/ Lists/Medias/marijuana-statement.pdf (accessed 2017 June 20).

4. Bill C-45: An Act respecting cannabis and to amend the Controlled Drugs and Substances Act, the Criminal Code and other Acts. 1st reading, 2017 Apr. 13, 1st Session, 42nd Parliament, 2017.

5. National Academies of Sciences, Engineering, and Medicine. The health effects of cannabis and cannabinoids: the current state of evidence and recommendations for research. Washington (DC): The National Academies Press; 2017.

6. Ligresti A, De Petrocellis L, Di Marzo V. From phytocannabinoids to cannabinoid receptors and endocannabinoids: pleiotropic physiological and pathological roles through complex pharmacology. Physiol Rev 2016;96:1593-659.

7. Maccarrone M, Bab I, Biro T, et al. Endocannabinoid signaling at the periphery: 50 years after THC. Trends Pharmacol Sci 2015;36:277-96.

8. Soethoudt M, Grether U, Fingerle J, et al. Cannabinoid CB2 receptor ligand profiling reveals biased signalling and off-target activity. Nat Commun 2017;8: 13958.

9. Pacher P, Kunos G. Modulating the endocannabinoid system in human health and disease - successes and failures. FEBS J 2013;280:1918-43.

10. Cohen PJ. Medical marijuana: the conflict between scientific evidence and political ideology. Part two of two. J Pain Palliat Care Pharmacother 2009;23:120-40.
Competing interests: Melanie Kelly is a director of and the Chief Scientific Officer for Panag Pharma, Halifax, NS. She has received nonfinancial support for conference travel and registration from the Natural Health Product Research Society of Canada, and she holds patent WO2015/074137. No other competing interests were declared.
This article has been peer reviewed.

Affiliations: Department of Pharmacology (Cairns, Kelly); Department of Anesthesia, Pain Management, and Perioperative Medicine (Kelly), Dalhousie University, Halifax, NS

Contributors: Both authors contributed substantially to the conception and design of the work, drafted the manuscript and revised it critically for important intellectual content, gave final approval of the version to be published and agreed to be accountable for all aspects of the work.

Correspondence to: Elizabeth Cairns, elizabeth.cairns@dal.ca 